\title{
Mycobacterium avium restriction fragment lenght polymorphism- IS IS1245 and the simple double repetitive element polymerase chain reaction typing method to screen genetic diversity in Brazilian strains
}

\author{
Patrícia Carvalho de Sequeira/ + , Leila de Souza Fonseca*, Marlei Gomes da Silva*, \\ Maria Helena Féres Saad ${ }^{+}$
}

Departamento de Micobacterioses, Instituto Oswaldo Cruz-Fiocruz, Av. Brasil 4365, 21045-900 Rio de Janeiro, RJ, Brasil

*Instituto de Microbiologia, Universidade Federal do Rio de Janeiro, Rio de Janeiro, RJ, Brasil

Simple double repetitive element polymerase chain reaction (MaDRE-PCR) and Pvu II-IS1245 restriction fragment length polymorphism (RFLP) typing methods were used to type 41 Mycobacterium avium isolates obtained from 14 AIDS inpatients and 10 environment and animals specimens identified among 53 mycobacteria isolated from 237 food, chicken, and pig. All environmental and animals strains showed orphan patterns by both methods. By $\mathrm{MaDRE-PCR}$ four patients, with multiple isolates, showed different patterns, suggesting polyclonal infection that was confirmed by RFLP in two of them. This first evaluation of MaDRE-PCR on Brazilian M. avium strains demonstrated that the method seems to be useful as simple and less expensive typing method for screening genetic diversity in M. avium strains on selected epidemiological studies, although with limitation on analysis identical patterns except for one band.

Key words: IS1254-restriction fragment length polymorphism - Mycobacterium avium - MaDRE-polymerase chain reaction nontuberculous mycobacteria - AIDS

Even in developed countries human mycobacterial infections have been defeating the XX century science technology since tuberculosis by Mycobacterium tuberculosis complex and disseminated infection by nontuberculous mycobacteria (NTM) has emerged as an important health problem associated to acquired immunodeficiency syndrome (AIDS). In developing countries, such as Brazil, were tuberculosis was never controlled, infection by NTM, of which mostly M. avium complex (MAC), has been identified in immune compromised patients (Ferreira et al. 2002).

In 1996, the treatment of human immunodeficiency virus (HIV) infection underwent considerable changes. Protease inhibitors and non-nucleoside analogue reverse transcriptase inhibitors became available for clinical practice, and more recently the fusion inhibitors, as part of a combination regimen of a highly active antiretroviral treatment (HAART) (Carpenter et al. 1996, Barbaro et al. 2005). However, although has been reported decrease of disseminated MAC (DMAC) infection in colonized individu-

Financial support: Faperj, CNPq (Milênio), the Johns Hopkins University (grant 1U19AI45432-01) awarded by the National Institutes of Health (US). This study contents are the sole responsibility of its authors and do not necessarily represent the official views of the NIH.

+ Corresponding author. E-mail: saad@ioc.fiocuz.br

${ }^{++}$Present address: Division of Infection Disease School of Public Healthy, University of California, Berkley, CA-94720, US

Received 8 June 2005

Accepted 9 November 2005 als that argue in favor of a HAART protective effect against DMAC, restoration of CD4 counts seems not to protect patients against MAC colonization (Gadelha et al. 2002).

The epidemiology of the M. avium infection is not well understood. In the last decade available molecular methods have been useful for strains differentiation and for the understanding of epidemiological transmission of this environmental pathogen. M. avium has been typed successfully by IS1245-restriction fragment length polymorphism (RFLP) and pulsed field gel electrophoresis (PFGE) (Arbeit \& Slutsky 1993, Guerrero et al. 1995, Devallois \& Rastogi 1997, Ritacco et al. 1998, Saad et al. 1999, Legrand et al. 2000). However these methods are time consuming and expensive, besides they need a lot of extracted DNA. Picardeau and Vincent (1996) have introduced a rapid and easy polymerase chain reaction (PCR) based typing method of $M$. avium by amplification of the sequence between the IS1245 and IS1311, both present in this species. Despite it has been described with discriminatory power similar to IS1245-RFLP there is still disagreement about its discriminatory power (Pestel-Caron et al. 1999, Yoder et al. 1999).

The aim of this study is to evaluate the genetic polymorphism of Brazilian M. avium isolated from clinical and environmental source by the simple double repetitive elements (MaDRE-PCR) and compared with the IS1245-RFLP. We further evaluated the ability of the simple and rapid MaDRE-PCR to identify polyclonal infection since multiple isolates from AIDS patients were available.

\section{MATERIALS AND METHODS}

The study included 41 MAC isolates obtained from 14 inpatients (Table) at Clementino Fraga Filho University 
Hospital, a Reference Center for AIDS in Rio de Janeiro. HIV seropositive status was determined by ELISA/Western blot (Organon Teknica, Boxtei, The Netherland/ Dupont, Welmington, US) and none of the patients was on HAART. All clinical information was obtained through retrospective review of the patients' medical records. A total of 237 environmental and animals specimens processed for culture in Löewenstein-Jensen at $37^{\circ} \mathrm{C}, 53$ yielded mycobacterial strains, of which ten (18.1\%) were identified as belonging to MAC; eight strains were obtained from swine lymph node living free in backyard and two from raw vegetable and chicken (slaughtered collected from abattoir), respectively. Species isolation, biochemical, and $M$. avium molecular identification by $247 \mathrm{bp}$ IS 1245-PCR fragments were performed as described previously (Ferreira et al. 2002, Leite et al. 2005).

Genotypic characterization of the samples with IS1245RFLP was performed as described by Saad et al. (1999). MaDRE-PCR was performed using a previously described method (Picardeau \& Vincent 1996) with minor modification. Briefly, total DNA was prepared by enzymatic protocol as described elsewhere (van Soolinger et al. 1991). Amplification reaction was performed by adding $49 \mu 1$ of Platinum ${ }^{\circledR}$ PCR Super Mix (LifeTechnologies, US) to $1 \mu 1$ of the extracted DNA, $0.5 \mathrm{M} \mathrm{MgCl}_{2}$ and $1 \mu \mathrm{M}$ of each specific oligonucleotide primers (PA: 5CAGAGCCTCA GGCGA3 and PB: 5CAGAGCCTCACGCGGA3 (LifeTechnologies). Initial $2 \mathrm{~min}$ denaturation at $95^{\circ} \mathrm{C}$ was followed by 35 amplification cycles of 1 min denaturation at $95^{\circ} \mathrm{C}, 1 \mathrm{~min}$ annealing at $55^{\circ} \mathrm{C}$ and 2 min elongation at $72^{\circ} \mathrm{C}$ and finally one cycle of $10 \mathrm{~min}$ extension at $72^{\circ} \mathrm{C}$ in MJ Research PTC-100 thermocycler (MJ Research Inc., MA, US). The amplicons were resolved on TBE $1.8 \%$ gel agarose containing $0.5 \mu \mathrm{g} / \mathrm{ml}$ ethidium bromide (Sigma \& Aldrich, Saint Louis, US) by $90 \mathrm{~V}$ eletrophoresis/2 h. Ladder $100 \mathrm{bp}$ was used as molecular weigh marker and $M$. avium clinical strain 40418 was used as standard isolate for all experiments.

DNA fingerprint pattern interpretation for both methods follow the convention established for genetic pattern generated for $M$. tuberculosis. MaDRE-PCR and RFLP banding pattern were analyzed visually. Strains with the same fingerprint from different patients were considered cluster and orphan patterns were those whose fingerprints were different from the others.

\section{RESULTS}

The strains were analyzed at first by $M a \mathrm{DRE}-\mathrm{PCR}$ and 41 human and ten environments isolates were amplified by PCR. The banding pattern revealed a maximum of eight bands, some of them intense and some other corresponding to weaker bands. None of the strains failed to be amplified and the patterns found were reproducible in two PCR performed in different days on the different DNA preparation. Eleven out of 14 patients had multiple isolates cultured at different or the same time as described in the Table. Most of the multiple isolates were obtained from sterile body sites $(27 / 38,71 \%)$, in $7 / 11$ patients all isolates showed the same MaDRE-PCR pattern for each patient (Fig. 1A). Patients 11 and 3 with three and five
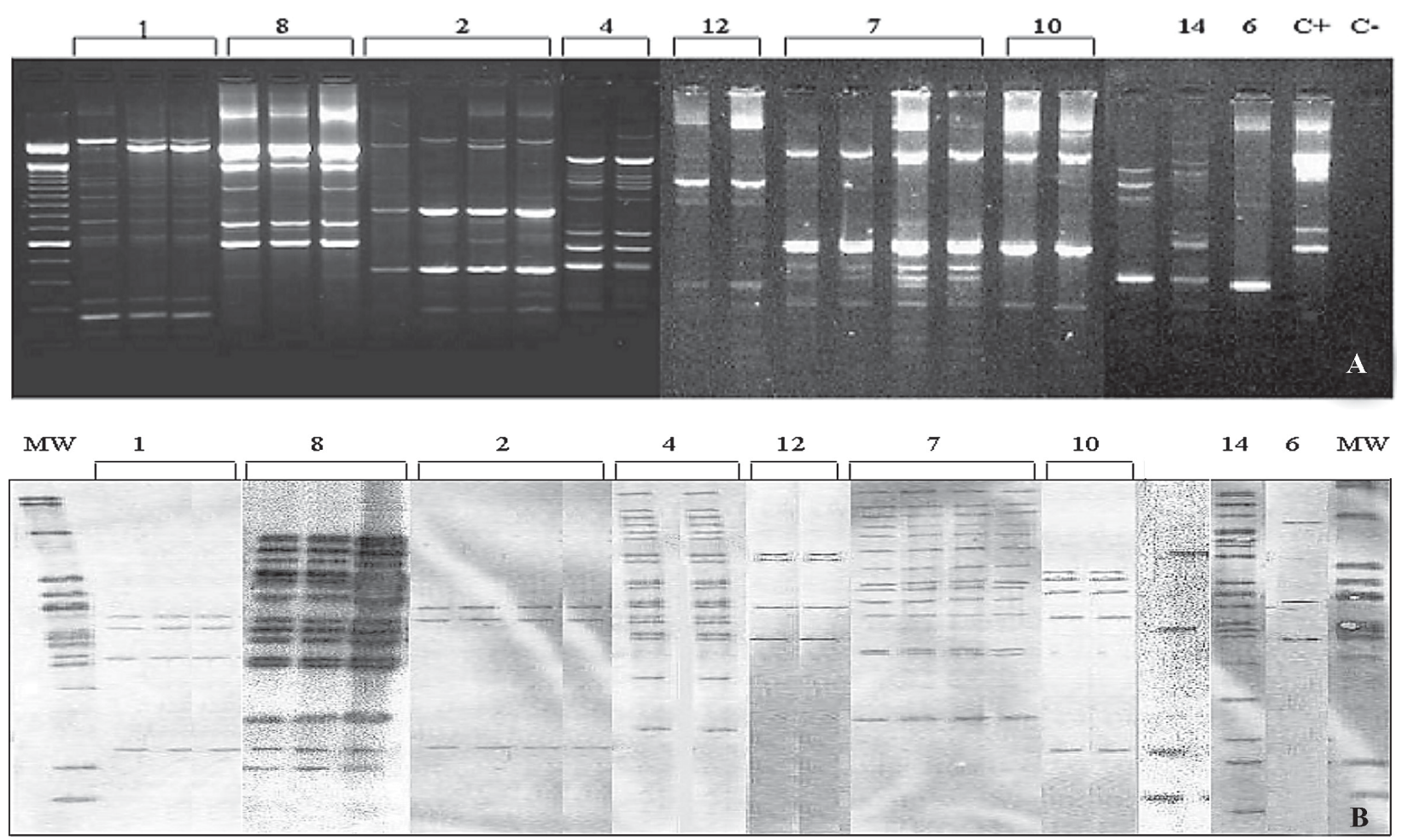

Fig. 1: Mycobacterium avium double repetitive elements-polymerase chain reaction (MaDRE-PCR) (A) and IS1245-restriction fragment lenght polymorphism (B) identical patterns of $M$. avium multiples isolates from Brazilian AIDS patients. Numbers are patients' identification. MW: molecular weigh; C+: M. avium standard strain 40418; C-: negative control; L: 100 bp ladder; 13, 14, and 6: patients with single isolates. 
multiple isolates, respectively, showed different MaDREPCR pattern in one of them (Fig. 2A). In the first patient two isolates were obtained from bronquialveolar lavage (BAL) and one from induced sputum; all were collected at the same day, however one BAL isolate had MaDRE-PCR banding pattern differing in number and position from the other isolates. The second patient had one isolate from non-sterile site (feces) and bone marrow, respectively, and three isolates from blood, all collected in different days. The bone marrow strain showed a MaDRE-PCR banding pattern differing in number and position from the other and it was isolated one month later (01/03/1997) from one of the blood isolates (12/09/1996) (Table). On the other hand, patients 5 and 9 , with three and four isolates each, obtained at the same or closer days, showed isolates with extra fragment despite of the majority of the bands being identical in size and position (Fig. 2B).

All strains from animals and vegetables yielded orphan $M a \mathrm{DRE}$ patterns and none were identical to human isolates (Fig. 3A).

RFLP polymorphism was complex showing three to 18 copies. Multiple isolates from different patients were found to be identical for each patient except for patients 3 and 11 confirming polyclonal pattern provided by $M a \mathrm{DRE}-$ PCR (Fig. 2A). However, for patients 5 and 9 RFLP isolates patterns were found to be identical (Fig. 2B).
For all multiple isolates from the same patients or from environment and animals MaDRE-PCR patterns interpretation was similar to that provided by RFLP. For all strains analyzed, cluster patterns were neither identified by PCR or RFLP.

\section{DISCUSSION}

This study performed with MaDRE-PCR was the first on Brazilian M. avium isolates and the results showed a limited number of bands (less than ten) as previously described by Pirardeau and Vincent (1996). Similar approach to type M. tuberculosis was used by Friedman et al. (1995) amplifying the nucleotides between the two repetitive elements; IS6110 and PGRS (G-C rich sequences). The reliability of the MaDRE-PCR typing method was confirmed by the same profile found on multiple isolates from the same patient. However, as described for M. tuberculosis DRE-PCR typing method, the presence of weak bands was observed. These features may result from cross hybridization with a related DNA sequence (Roiz et al. 1995, Picardeau \& Vincent 1996) or it is related to technical limitation of the method. The same explanation may be applied for the finding of multiple isolates from the same patients (5 and 9) with identical patterns except for one or two extra bands in one of the strains of each patient, but RFLP provided identical pattern. This may introduce some
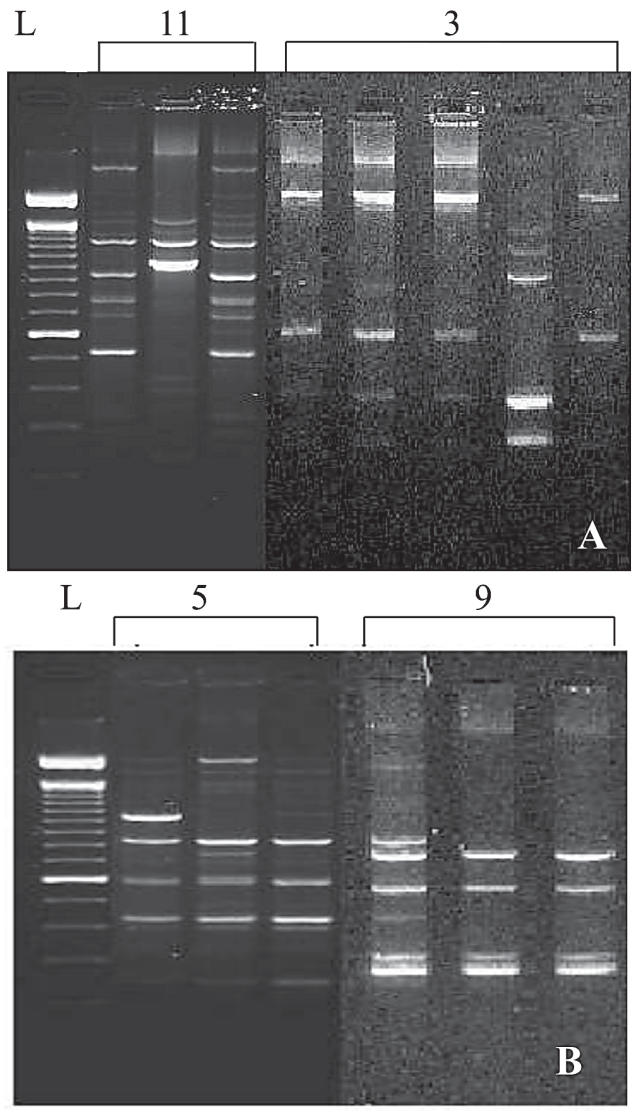

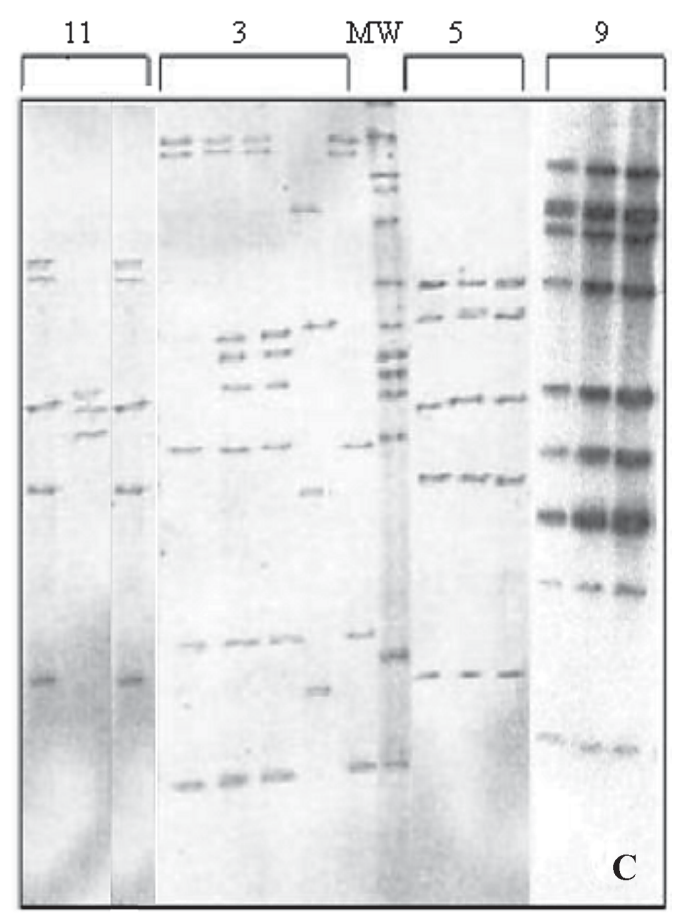

Fig. 2A, B: Mycobacterium avium double repetitive elements-polymerase chain reaction (MaDRE-PCR) results on multiple isolates from patients $11,3,5$, and 9 showing different banding pattern in one of the isolates. C: IS1245-restriction fragment length polymorphism confirming polyclonal patterns in isolates from patients 11 and 3, and identical patterns for isolates from patients 5 and 9 . L: 100 bp ladder; MW: molecular weigh. 
difficulty for MaDRE pattern interpretation. However, where PCR amplification strains produced complete different patterns also displayed unrelated RFLP profiles (patients 3 and 11).

PCR and RFLP in patient 3, both discriminate infection involving different $M$. avium strains. In fact this patient had seven isolates during a period of 6 months and we analyzed five of those. Our result characterized polyclonal infection with persistence of the initial strain in blood. Picardeau and Vincent (1996) observed similar finding, when they studied sequential strains of $M$. avium isolated over 23 months. Despite similar results have been observed for patient 11 polyclonal infection could not be characterized. Human NTM infection is defined by a minimum criteria such as: (a) repeated isolates of the same strains from natural clinical specimen from sterile site with moderate to intense in vitro culture growth; (b) clinical evolution of active disease; and (c) exclusion of other pathogenic agent (Wallace et al. 1990). Contrarily to patient 3, patient 11 did not fulfill these criteria. Furthermore, strains obtained from non-sterile site may be only a colonize event and NTM, as M. avium, can be isolate from respiratory tract from healthy patients (Portaels 1995).

The overall result of PCR typing based on two insertion sequences present in $M$. avium provided a discriminatory power similar to RFLP results, since in both methods most of related strains yielded identical patterns and unrelated strains produced different profile, but careful interpretation must be taken among strains closely related, with identical banding pattern exception for one extra band. This finding is in agreement with results obtained by other comparing PCR-typing and PFGE methods (Pestel-Caron et al. 1999).

In both molecular typing system no cluster strains was seen in human, animals, and environment strains. Exposure to MAC organisms is impossible to avoid because they are ubiquitous, and the human infection reservoir remains unclear. Hot water (von Reyn et al. 1994), soil (Eaton et al. 1995), and animals such as pig have been proposed to be a source of $M$. avium for human infection. However Brazil does not have such hot water system and isolation of MAC strains from soil is a difficult task. Has been described that MAC pigs, food, and human isolates shared high degree of RFLP similarity, but direct link between them was not found (von Reyn et al. 1994, Mansfield \& Lackner 1997, Yoder et al. 1999). Our results showed low genotyping patterns similarities between humans, environments, and animals strains but our sampling is too small and the environmental and animals' specimens were not taken from the same patients' geographic area. PCR typing to screening diversity of $M$. avium infecting human may favor links between environmental and animals' sources in further epidemiologal studies.

In summary, this study shows that the rapid, easy, and inexpensive PCR typing based on two M. avium-insertion sequences is useful for epidemiological studies such
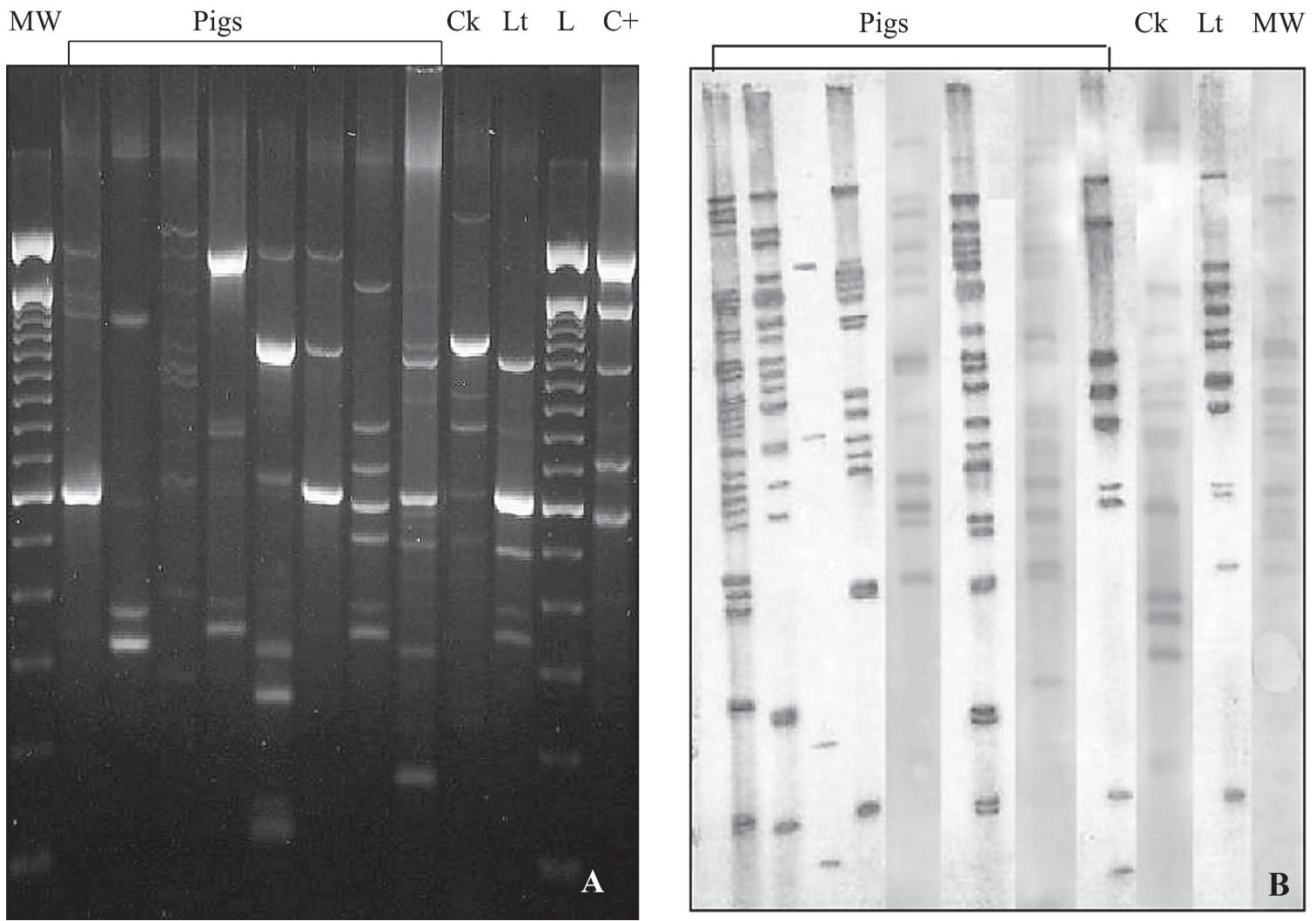

Fig. 3: Mycobacterium avium double repetitive elements-polymerase chain reaction (MaDRE-PCR) (A) and IS1245-restriction fragment length polymorphism (B) patterns isolates from pigs, chicken (Ck), and lettuce (Lt). L: 100 bp ladder; C+: M. avium strain 40418; MW: molecular weigh. 
TABLE

Source of Mycobacterium avium isolates from 14 acquired immunodeficiency syndrome Brazilian patients

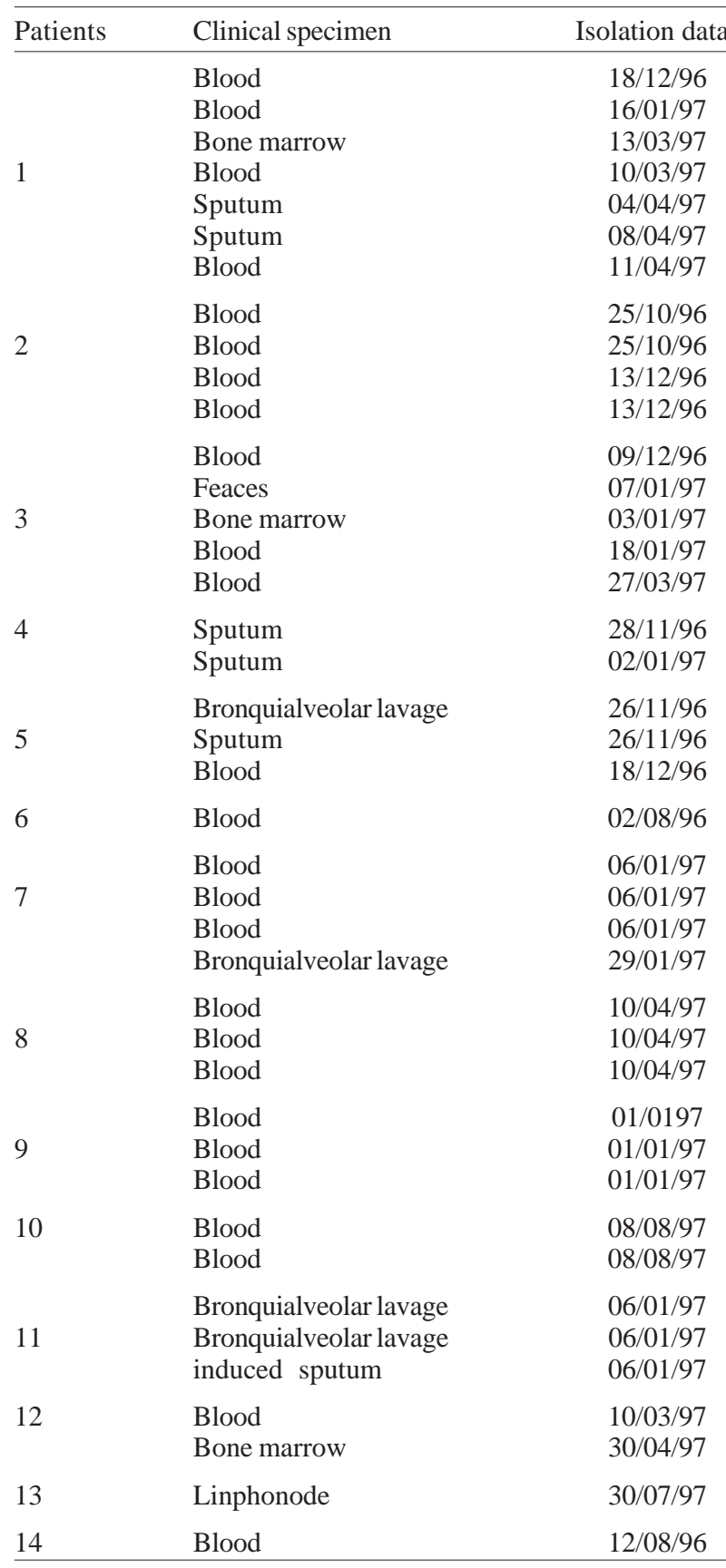

as detection of polyclonal infection, an event that may have treatment implications (Arbeit et al. 1993, Saad et al. 2000, Panunto et al. 2003). However we suggest to strict its use in small sampling from select set because of the limitation on analysis of patterns with difference in one band.

Despite HAART has dramatically decreased $M$. avium infections, it would be useful to have a simple and inexpensive genotypic method to easily screen a possible emergency of new genotype of $M$. avium among HIV patients with a change in their immunological status related to begging HAART (detection of a particular genotype in preexisting polyclonal infection) or when preexisting $M$. avium suppressive therapies or prophylaxis in patients on HAART are withdrawn (Legrand et al. 2000) or to follow the epidemiological evolution in HAART patient that remains colonized by $M$. avium as showed by Gadelha et al. (2002).

\section{REFERENCES}

Arbeit RD, Slutsky A 1993. Genetic diversity of M. avium strains causing monoclonal and polyclonal bacteremia in patients with AIDS. J Infect Dis 167: 1384-1390.

Barbaro G, Scozzafava A, Mastrolorenzo A, Supuran CT 2005. Highly active antiretroviral therapy; current state of the art, new agents and their pharmacological interactions useful for improving therapeutic outcome. Curr Pharm Des 11: 1805-1843.

Carpenter JCC, Fischl AM, Hammer MS 1996. Antiretroviral therapy for HIV infection in 1996. Recommendations of the International AIDS Society, USA panel. JAMA 276: 7886.

Devallois A, Rastogi N 1997. Computer-assisted analysis of Mycobacterium avium fingerprints using insertion elements IS1245 and IS1311 in a Caribbean setting. Res Microbiol 14: 703-13.

Eaton T, Falkinham JO, Aisu TO, Daniel TM 1995. Isolation and characteristics of Mycobacterium avium complex from water and soil samples in Uganda. Tuber Lung Dis 76: 570574.

Ferreira RM, Saad MH, Silva MG, Fonseca L de S 2002. Nontuberculous mycobacteria I: one year clinical isolates identification in Tertiary Hospital Aids Reference Center, Rio de Janeiro, Brazil, in pre highly active antiretroviral therapy era. Mem Inst Oswaldo Cruz 97: 725-729.

Friedman CR, Stoeckle MY, Johnson WD, Riley LW 1995. Double-repetitive-element PCR method for subtyping $M y$ cobacterium tuberculosis clinical isolates. J Clin Microbiol 33: 1383-1384.

Gadelha A, Accacio N, Grinzstejn B, Veloso V, da Silveira LB, Fandinho F, Saad MH, Lourenço MC, Rolla V 2002. Low incidence of colonization and no cases of disseminated $M y$ cobacterium avium complex infection (DMAC) in Brazilian AIDS patients in the HAART era. Braz J Infect Dis 6: 252-257.

Guerrero C, Bernasconi C, Burki D, Bodmer T, Telenti A 1995. A novel insertion element from Mycobacterium avium, IS1245, is a specific target for analysis of strain relatedness. J Clin Microbiol 33: 304-3077.

Legrand E, Sola C, Verdol B, Rastogi N 2000. Genetic diversity of Mycobacterium avium recovered from AIDS patients in the Caribbean as studied by a consensus IS1245-RFLP method and pulsed-field gel electrophoresis. Res Microbiol 151: 271-283.

Leite CQ, da Silva Rocha A, de Andrade Leite SR, Ferreira RM, Suffys PN, de Souza Fonseca L, Saad MH 2005. A comparison of mycolic acid analysis for nontuberculous mycobacteria identification by thin-layer chromatography and molecular methods. Microbiol Immunol 49: 571-578. 
Mansfield KG, Lackner AA 1997. Simian immunodeficiency virus-inoculated macaques acquire Mycobacterium avium from potable water during AIDS. J Infect Dis 175: 184-187.

Panunto AC, Villares MC, Ramos MC 2003. IS1245 restriction fragment length polymorphism typing of Mycobacterium avium from patients admitted to a reference hospital in Campinas, Brazil. Braz J Med Biol Res 36: 1397-1401.

Pestel-Caron M, Graff G, Berthelot G, Pons JL, Lemeland JF 1999. Molecular analysis of Mycobacterium avium isolates by using pulsed-field gel electrophoresis and PCR. $J$ Clin Microbiol 37: 2450-2455.

Picardeau M, Vincent V 1996. PCR typing of M. avium isolates. J Clin Microbiol 34: 389-392.

Portaels F 1995. Epidemiology of mycobacterial disease. Clin Dermat 13: 207-222.

Roiz MP, Palenque E, Guerrero C, Garcia MJ 1995. Use of restriction fragment length polymorphism as a genetic marker for typing M. avium strains. Clin Microbiol 33: 1389-1391.

Ritacco V, Kremer K, van der Laan T, Pijnenburg JE, de Haas PE, van Soolingen D 1998. Use of IS901 and IS1245 in RFLP typing of Mycobacterium avium complex: relatedness among serovar reference strains, human and animal isolates. Int J Tuberc Lung Dis 2: 242-251.

Saad MH, Fonseca LD, Ferrazoli L, Fandinho F, Palaci M,
Grinsztejn B, Kritski A, Werneck A, Poltoratskaia N, Johnson Jr WD, Riley LW 1999. IS1245 genotypic analysis of Mycobacterium avium isolates from patients in Brazil. Int J Infect Dis 3: 192-196.

Saad MH, Telles MA, Porfirio F, Ferrazoli L, de Souza Fonseca L, Johnson Jr W, Riley LW 2000. Multiple isolates from Aids patients; aspects of an analysis by a genotypic marker and antimicrobial susceptibilities variations. Mem Inst Oswaldo Cruz 95: 729-32.

van Soolingen D, Hermans PW, Haas PEW, Soll DR, Van Embden JDA 1991. Occurrence and stability of insertion sequences in Mycobacterium tuberculosis complex strains: evaluation of an insertion sequence-dependent DNA polymorphism as a tool in the epidemiology of tuberculosis. $J$ Clin Microbiol 29: 2578-2586.

von Reyn CF, Maslow JN, Barber TW, Falkinham III JO, Arbeit RD 1994. Persistent colonization of potable water as a source of M. avium infection in AIDS patients. Lancet 343: $1137-1141$.

Yoder S, Argueta C, Holtzman A, Aronson T, Berlin OG, Tomasek P, Glover N, Froman S, Stelma Jr G 1999. PCR comparison of Mycobacterium avium isolates obtained from patients and foods. Appl Environ Microbiol 65: 2650-2653.

Wallace RJ, O’Braien R, Glassroth J, Raleigh J, Dutt A 1990 Diagnosis and treatment disease caused by non tuberculous mycobacteria. Am Rev Respir Dis 142: 149. 